\title{
Relevant Aspects of the Allotriomorphic and Idiomorphic Ferrite Transformations Kinetics
}

\author{
C. Capdevila, F. G. Caballero and C. García de Andrés*
}

Department of Physical Metallurgy, Centro Nacional de Investigaciones Metalúrgicas (CENIM) Consejo Superior de Investigaciones Científicas (CSIC), Avda. Gregorio del Amo, 8. 28040 Madrid, Spain

* Corresponding author. Tel.: 00-34-915538900; fax: 00-34-915347425

E-mail address: cgda@cenim.csic.es (C. García de Andrés) 


\begin{abstract}
Recently, the grain boundary-intragranular nucleation of ferrite has received great attention seeking for the improvement of the toughness in the steel, specially when conventional austenite grain refinement techniques are not enough. This paper deals with some relevant aspects about the competitive process of allotriomorphic and idiomorphic ferrite transformations in a medium carbon vanadium-titanium microalloyed steel for two different isothermal temperatures and different values of prior austenite grain sizes. It is concluded that the consideration of proeutectoid ferrite (allotriomorphic + idiomorphic) as a single transformation might lead to wrong conclusions in an analysis of the isothermal decomposition of austenite.
\end{abstract}




\section{Introduction}

As a result of a survey of the behaviour of plain carbon steels, Dubé ${ }^{1}$ and Aaronson ${ }^{2}$ proposed a classification of morphologies of pro-eutectoid ferrite which occur as the $\gamma / \alpha$ transformation temperature is lowered. They recognised two well-defined morphologies of ferrite which grow by a diffusional mechanism: allotriomorphic ferrite and idiomorphic ferrite. The former nucleates at the prior austenite grain boundaries, meanwhile the latter usually forms intragranularly at inclusions. ${ }^{3-5}$

Toughness and other mechanical properties of steels are strongly affected by their microstructure. A microstructure formed by ferrite nucleated at both the austenite grain surfaces and intragranular sites, increases the difficulty of the propagation of cleavage cracks ${ }^{6}$. Recently, this phenomenon has received great attention seeking for the improvement of the toughness in the steel, specially when conventional austenite grain refinement techniques are not enough. ${ }^{7-12}$ Therefore, a thorough study dealing with the simultaneous transformation of austenite in allotriomorphic and/or idiomorphic ferrite is vital.

Authors have reported recently studies about the kinetics and mechanisms of allotriomorphic ${ }^{13-15}$ and idiomorphic ${ }^{16}$ isothermal ferrite formation in a medium carbon vanadium-titanium microalloyed steel. In the present work, relevant aspects of both transformations have been presented.

\section{Experimental Techniques}


The chemical composition of the steel studied is presented in Table 1 . The material was supplied in the form of $50 \mathrm{~mm}$ square bars, obtained by conventional casting as a square ingot (2500 kg) and hot rolling to bar.

(Table 1)

The isothermal decomposition of austenite has been analysed by means of a highresolution dilatometer DT 1000 Adamel-Lhomargy described elsewhere ${ }^{17}$. Cylindrical dilatometric samples of $2 \mathrm{~mm}$ in diameter and $12 \mathrm{~mm}$ in length, machined parallel to the rolling direction of the bar, were used for these tests. The change in length of the specimen is transmitted via amorphous silica push rod. This variation is measured by a linear variable differential transformer $(L V D T)$ sensor in a gas-tight enclosure enabling testing under vacuum or an inert atmosphere with accuracy better than $0.1 \mu \mathrm{m}$. The dilatometric curve (relative change in length $\left(d L / L_{o}\right)$ vs. time $\left.(t)\right)$ is monitored with the help of a computer assisted electronic device. The dilatometer is equipped with a very low thermal inertia radiation furnace. The heat radiated by two tungsten filament lamps is focused on the specimen by means of a bi-elliptical reflector. The temperature is measured with a $0.1 \mathrm{~mm}$ diameter Chromel - Alumel (Type K) thermocouple welded to the specimen. Cooling is carried out by blowing a jet of helium gas directly onto the specimen surface. The helium flow-rate during cooling is controlled by a proportional servo valve. Heating and cooling devices of this dilatometer ensure an excellent efficiency in controlling the temperature and holding time of isothermal treatments, and fast cooling in quenching processes. 
With the aim of studying the influence of PAGS on the isothermal austenite-toallotriomorphic and idiomorphic ferrite transformation, specimens were austenitised at two different temperatures (1273 K and $1523 \mathrm{~K}$ ) for $1 \mathrm{~min}$. Subsequently, specimens were isothermally transformed at 913 and $873 \mathrm{~K}$ during different times, and quenched under helium gas flow at a cooling rate of $200 \mathrm{~K} / \mathrm{s}$. Specimens were ground and polished using standardised metallographic techniques, and subsequently etched with 2pct-Nital solution to reveal the ferrite microstructure by optical microscopy.

The PAGS measurements were made on micrographs. The average grain size was calculated by using a linear intercept technique involving at least 50 intercepts, permitting the count of the number of grains intercepted by the grid line. The effects of a moderately non-equiaxed structure may be eliminated by counting the intersections of lines in four or more orientations covering all the observation fields with an approximately equal weight ${ }^{18}$. Table 2 shows the average PAGS in microns corresponding to both austenitisation conditions.

\section{(Table 2)}

Measurements of volume fraction of allotriomorphic $\left(\xi_{\alpha}\right)$ and idiomorphic ( $\left.\xi_{I D I}\right)$ ferrite were performed statistically by a systematic manual point counting procedure ${ }^{18}$. A grid superimposed on recorded micrographs provides, after a suitable number of placements, an unbiased statistical estimation of $\xi_{\alpha}$ and $\xi_{I D I}$.

Finally, the morphology and nature of the inclusions were determined by means of scanning electron microscopy (SEM). Specimens were sectionalised longitudinally and 
transversally to the rolling direction, polished in the usual way and finished on $0.5 \mu \mathrm{m}$ diamond paste. Samples were slightly etched in a 2 pct-Nital solution for SEM examination in a JEOL JXA 840 scanning electron microscope operating at $15 \mathrm{kV}$ with an energy disperse X-ray analysis unit. Quantitative information on the size distribution of inclusions was obtained by means of an IBAS OPTIMAS 2.0 automatic image analyser. The size of the inclusions was determined through the measurement of the two perpendicular diameters in the transversal section $\left(d_{1}\right.$ and $\left.d_{2}\right)$, and the highest diameter in the longitudinal section $\left(d_{3}\right)$. For that propose, a $512 \times 512$ points grid was used along with magnifications as high as 3000 times to characterise inclusions with a diameter lower than $0.1 \mu \mathrm{m}$. Minimum of 500 particles were counted to obtain a reliable result.

\section{Results and discussion}

\subsection{Size distribution and nature of inclusions}

EDX spectrum in Fig. 1 shows that all the inclusions which are present in the steel are MnS. This figure also shows the morphology of the inclusions in samples sectionalised longitudinally and transversally to the rolling direction, and the frequency of the inclusion diameters experimentally obtained in the transversal $\left(d_{1}\right.$ and $\left.d_{2}\right)$ and longitudinal $\left(d_{3}\right)$ sections. From the histograms of this figure, the average values of $d_{1}$ $=1.78 \mu \mathrm{m}, d_{2}=1.16 \mu \mathrm{m}$, and $d_{3}=9.56 \mu \mathrm{m}$ are obtained.

Idiomorphic ferrite nucleates intragranularly in the inclusions distributed inside the austenite grains. Thus, the volume fraction of idiomorphic ferrite is related to the volume 
fraction of inclusions in the steel. Kluken and Grong ${ }^{19}$ proposed an equation to estimate the volume fraction of inclusions by converting the analytical oxygen and sulphur concentration in the steel into an equivalent inclusion volume fraction. Considering the solubility of sulphur in the steel equal to $0.003{\mathrm{wt}-\mathrm{pct}^{19}}$, the following equation has been derived:

$V_{V} \approx 10^{-2}[5.0\{\% O\}+5.4(\{\% S\}-0.003)]$

where $V_{V}$ is the volume fraction of inclusions in the steel and $O$ and $S$ are the oxygen and sulphur concentration of the steel, respectively, in wt-pct. $V_{V}=2.34 \times 10^{-3}$ for the studied steel (See Table 1 for oxygen and sulphur composition).

\subsection{Role of PAGS on the competitive allotriomorphic and idiomorphic ferrite formation}

Since allotriomorphic ferrite nucleates at the prior austenite grain boundaries and idiomorphic ferrite nucleates at the inclusions inside the austenite grains, the balance between the number of intragranular nucleation sites and those at the austenite grain boundaries is a very important factor in the competitive process of allotriomorphic idiomorphic ferrite formation.

It is well known that an increase in the PAGS leads to a reduction in the number of nucleation sites at the austenite grain boundaries. Therefore, an increase in the PAGS indirectly favours the intragranular nucleation of ferrite, and then the formation of idiomorphic ferrite is enhanced instead of allotriomorphic ferrite. Likewise, as the PAGS 
increases, the number of inclusions trapped inside the austenite grains increases, promoting the intragranular nucleation of ferrite.

Assuming that ferrite nucleates primarily on grain boundaries, Grong ${ }^{20}$ reported that the total number of grain boundary nucleation sites per unit volume, $N_{V}^{G B}$ is given as:

$N_{V}^{G B}=n_{a} S_{V}^{G B}$

where $n_{a}$ is the number of nucleation sites per unit grain boundary area and $S_{V}^{G B}$ is the grain boundary surface area per unit volume. The value of $n_{a}$ can be expressed as $K / \delta^{2}$ where $K$ is a constant and $\delta$ is the atomic spacing (reasonable value of $\left.2.5 \times 10^{-10} \mathrm{~m}\right)^{21}$. As a second approximation, it could be also assumed the austenite grains are tetrakaidecahedra. Then, $S_{V}^{G B}$ is given as: $:^{13,22}$

$$
S_{V}^{G B}=\frac{3.34}{d_{\gamma}}
$$

where $d_{\gamma}$ is the mean austenite grain diameter.

On the other hand, the total inclusion surface area per unit volume, $S_{V}^{I N C}$, could be expressed by:

$S_{V}^{I N C}=S_{V o}^{I N C} n^{I N C}$ 
where $S_{V o}^{I N C}$ is the inclusion surface area per unit volume for a particular inclusion, and $n^{I N C}$ is the total number of inclusions inside the austenite grain. In this sense, and considering that all the inclusions are ellipsoids with the same size, $S_{V o}^{I N C}$ is written as:

$S_{V o}^{I N C}=6 / d_{3}$

Moreover, $n^{I N C}$ could be expressed as the volume fraction of inclusions in the steel $\left(V_{V}\right)$ modulated by the ratio between the austenite grain size and the inclusion size,

$n^{I N C}=\frac{d_{\gamma}}{\left(d_{1}+d_{2}\right) / 2} V_{V}$

Therefore, $S_{V}^{I N C}$ can be calculated as follows:

$S_{V}^{I N C}=\frac{6}{d_{3}} \frac{d_{\gamma}}{\left(d_{1}+d_{2}\right) / 2} V_{V}=\frac{12 d_{\gamma}}{d_{3}\left(d_{1}+d_{2}\right)} V_{V}$

The intragranular nucleation sites per unit volume, $N_{V}^{I N C}$, could be expressed by:

$$
N_{V}^{I N C}=n_{i} S_{V}^{I N C}
$$


where $n_{i}$ is the number of nucleation sites per unit surface area of inclusion which can be expressed as $K^{*} / \delta^{2}$ where $K^{*}$ is a constant and $\delta$ is the atomic spacing $\left(\delta=2.5 \times 10^{-10}\right.$ $\mathrm{m})^{21}$.

Figure 2 shows a comparison between equation (3) and (7) which allows us to analyse the evolution of the grain boundary and intragranular nucleation sites as PAGS increases. It is follow from this figure that intergranular nucleation sites become more important as PAGS increases.

\subsection{Kinetics of allotriomorphic and idiomorphic ferrite transformation}

The theoretical determination of the diffusivity of carbon in austenite, $D_{C}^{\gamma}$, performed by Siller and McLellan ${ }^{23}$ and reviewed by Bhadeshia, ${ }^{24}$ considers both the kinetic and equilibrium thermodynamic behaviour of carbon in austenite. Calculation of $D_{C}^{\gamma}$ takes also into account two important factors: the concentration dependence of the activity of carbon in austenite and the repulsive interactions between nearest neighbouring carbon atoms located in octahedral interstitial sites. Thus, the diffusivity $D_{C}^{\gamma}$ is calculated by two factors, a concentration dependent factor and a concentration independent factor,

$$
D_{C}^{\gamma}=\xi(\theta) \frac{k_{B} T}{h}\left(\frac{\lambda^{2}}{3 \gamma_{m}}\right) \exp \left\{-\frac{\Delta G^{*}}{k_{B} T}\right\}
$$


where $\xi(\theta)$ is the carbon concentration dependent factor obtained according to Bhadeshia's calculations $^{24}$ and takes values listed in Table 3; $\Delta G^{*}$ is the activation free energy for diffusion which is independent of composition and temperature; $\gamma_{m}$ is an activity coefficient assumed constant; $\lambda$ is the distance between the $\{002\}$ austenite planes and $h$ is the Planck's constant. Bhadeshia ${ }^{24}$ found that $\Delta G^{*} / k_{B}=21230 \mathrm{~K}$ and $\ln \left\{\gamma_{m} / \lambda^{2}\right)=31.84$. Likewise, Babu and Bhadeshia ${ }^{25}$ completed this model analysing the effect of different substitutional alloy elements on the diffusivity of carbon in austenite. The values of $D_{C}^{\gamma}$ for temperatures of 913 and $873 \mathrm{~K}$ are listed in Table 3.

Reed and Bhadeshia ${ }^{26}$ described a theory which is able to model the nucleation and growth of allotriomorphic ferrite. The allotriomorphs, before site saturation, are considered as discs having their faces parallel to the austenite grain boundary plane. The discs are assumed to grow radially, and the half thickness $Z$ and radius $\eta Z$, being $\eta$ the aspect ratio of the allotriomorphs, varies parabolically with time, through the equation $Z=\alpha_{1} t^{1 / 2}$ where $\alpha_{1}$ is the one - dimensional parabolic growth rate constant and $t$ represents the growth time. The aspect ratio $\eta$ of the allotriomorphs is considered constant, because the lengthening and thickening processes are actually coupled. Consistent with experimental evidences, ${ }^{27} \eta$ is assumed to have a value of 3 . The value of $\alpha_{1}$ can be obtained by numerical solution from the equation, ${ }^{28}$

$$
\alpha_{1} \exp \left(\frac{\alpha_{1}{ }^{2}}{4 D_{C}^{\gamma}}\right) \operatorname{erfc}\left(\frac{\alpha_{1}}{2 \sqrt{D_{C}^{\gamma}}}\right)=2\left(\frac{D_{C}^{\gamma}}{\pi}\right)^{1 / 2} \frac{C^{\gamma \alpha}-\bar{C}}{C^{\gamma \alpha}-C^{\alpha \gamma}}
$$


where $D_{C}^{\gamma}$ is the diffusivity of carbon in austenite, $\bar{C}$ is the overall carbon content, $C^{\gamma \alpha}$ is the austenite solute content at the interface, and $C^{\alpha \gamma}$ is the ferrite solute content at the interface. According to Bhadeshia, ${ }^{29}$ the consideration of paraequilibrium is a good approach to the modelling of the kinetics of this transformation. In that case, partitioning of substitutional solute atoms is not able to occur and the adjoining phases have identical $\mathrm{X} / \mathrm{Fe}$ atom ratios, where $\mathrm{X}$ represents the substitutional solute elements. The substitutional atoms remain configurationally frozen, but interstitial solutes such as carbon are able to partition and attain equilibration of chemical potential in both phases. Hence, the values of $C^{\gamma \alpha}$ and $C^{\alpha \gamma}$ in Eqn. (9) refer to carbon concentrations, and they were calculated according to the procedure reported by Shiflet et al. ${ }^{30} C^{\gamma \alpha}$ and $C^{\alpha \gamma}$ values as well as $\alpha_{1}$ values are listed in Table 3.

On the other hand, idiomorphic ferrite nucleates on the MnS inclusions distributed inside the austenite grain. Therefore, its growth is modelled as a spherical particle nucleated in the inclusion and growing inside the austenite grain. The three-dimensional parabolic thickening constant $\left(\alpha_{3}\right)$ is given by: ${ }^{28}$

$$
\alpha_{3}=\left[\frac{2 D_{C}^{\gamma}\left(\bar{C}-C^{\alpha \gamma}\right)}{C^{\gamma \alpha}-\bar{C}}\right]^{1 / 2}
$$

The values of $\alpha_{3}$ for temperature of 913 and $873 \mathrm{~K}$ are listed in Table3.

(Table 3) 


\subsection{Comparison between allotriomorphic and idiomorphic ferrite overall} transformation kinetics

The evolution of the volume fraction of allotriomorphic ferrite $\left(\xi_{\alpha}\right)$ with time during the isothermal decomposition of austenite could be calculated within the framework of Johnson-Mehl-Avrami heterogeneous transformation kinetics theory as was extensively reported by the authors in Refs.13-15, and it is given by:

$$
\xi_{\alpha}=\xi_{\alpha}^{E Q}\left[1-\exp \left(-\frac{2 S_{V}^{G B} \alpha_{1} t^{1 / 2} I(t)}{\phi}\right)\right]
$$

where $\xi_{\alpha}^{E Q}$ is the equilibrium (or maximum) volume fraction of allotriomorphic ferrite isothermally formed at a given temperature, $\alpha_{1}$ is the parabolic growth rate constant for planar growth, and $t$ is the isothermal holding time which is defined to be zero as the allotriomorphic ferrite starts forming after an incubation period. The process of nucleation is taking into account in the equation by means of the function $I(t)$, which takes values between 0 and 1 . A value of $I(t)=0$ means that nucleation has not started. By contrast, a value of $I(t)=1$ means that saturation of nucleation sites has been reached. The expression for $I(t)$ is described in Refs.14. The parameter $\phi$ is the supersaturation in carbon, which can be estimated from the phase diagram as described in Ref.. Values of all of these parameters for the two studied isothermal temperatures, are listed in Table 4. Finally, the density of nucleation sites in the austenite grain 
boundary is taking into account in equation (11) by means of equation (3). The values of $S_{V}^{G B}$ for the two PAGS considered in this study are listed in Table 4.

\section{(Table 4)}

On the other hand, idiomorphic ferrite nucleates on inclusions randomly distributed in the austenite phase at a constant nucleation rate $\left(I_{I D I}\right)$, and subsequently, grows isotropically to form spherical particles. The classical Johnson-Mehl-Avrami equation can be applied to describe the evolution of the volume fraction of idiomorphic ferrite $\left(\xi_{I D I}\right)$ with time, and the following expression was derived:

$$
\xi_{I D I}=\xi_{I D I}^{E Q}\left[1-\exp \left(-\frac{8 \pi}{15} I_{I D I} S_{V}^{I N C} \alpha_{3}^{3} t^{5 / 2}\right)\right]
$$

where $\xi_{I D I}^{E Q}$ is the equilibrium (or maximum) volume fraction of idiomorphic ferrite isothermally formed at a given temperature, $\alpha_{3}$ is the parabolic thickening rate constant for isotropic growth, $t$ is the isothermal holding time, and $S_{V}^{I N C}$ is the total inclusion surface area per unit volume (equation (7)).

The nucleation rate, $I_{I D I}$, has been calculated according to Lange et $a l .{ }^{31}$ and Reed and Bhadeshia, taking into account the chemical composition of the studied steel. The values of $S_{V}^{I N C}$ for the two PAGS studied and the parameters included in equation (7) for a temperature of 913 and $873 \mathrm{~K}$ are listed in Table 5. 
The theory takes into account the hard impingement between allotriomorphs growing from the same, or adjacent, austenite grain boundaries. It should be notice, however, that soft-impingement (the overlap of diffusion fields) between neighbouring allotriomorphs is ignored. This is a good approximation if the isothermal temperature is lower that the temperature at which pearlite start to form $\left(A e_{1}\right)$. At temperatures lower than $A e_{1}$, the isothermal decomposition of austenite yields to a ferrite-pearlite final microstructure. Pearlite is a lamellar product of eutectoid decomposition. A pearlite nodule is composed of multiple colonies; each colony has parallel lamellae of ferrite and cementite, which are orientated differently with respect to lamellae in adjacent colonies. The austenite transforms into pearlite by a reconstructive mechanism at temperatures below the eutectoid temperature $A e_{1}$. The formation of ferrite enriches in carbon the surrounding austenite promoting the formation of cementite nucleus at the $\alpha / \gamma$ interface and the local reduction of carbon content in the austenite that surrounds the cementite nucleus leads to the formation of ferrite. The ferrite and cementite formation process yields to the characteristic lamellar structure of pearlite. Since pearlite formation, the carbon enrichment of austenite due to the previous proeutectoid ferrite formation is avoided and carbon concentration in austenite far from the $\alpha / \gamma$ interface remains the same as the overall carbon content of the steel. Hence, soft-impingement effect could be neglected. In the studied steel, allotriomorphic and idiomorphic ferrite as well as pearlite are the phases obtained by the total decomposition of austenite at both temperatures. Therefore, with the aim of analysing the competitive transformation kinetics of allotriomorphic and 
idiomorphic ferrite, $\xi_{\alpha}$ and $\xi_{I D I}$ have been normalised by their respective equilibrium volume fractions ( $\xi_{\alpha}^{E Q}$ and $\left.\xi_{I D I}^{E Q}\right)$ :

$$
\xi^{*}=\frac{\xi_{\alpha} / \xi_{\alpha}^{E Q}}{\xi_{I D I} / \xi_{I D I}^{E Q}}
$$

The effect of the isothermal decomposition temperature on $\xi^{*}$ value is shown in Fig. 3. Figure 3(a) shows the evolution of $\xi^{*}$ during the first stages of transformation. The maximum in this figure suggests that the formation of allotriomorphic ferrite is faster than idiomorphic ferrite formation during the earliest stages of transformation. This is consistent with the fact that the lengthening processes of allotriomorphic ferrite along the grain boundaries occurs very fast producing a substantial increase on allotriomorphic ferrite volume fraction during the first stages of austenite decomposition. This phenomenon is shown in the microstructures of Fig. 4. Figure 4(a) shows the microstructure obtained after $60 \mathrm{~s}$ of isothermal decomposition of austenite (PAGS of 76 $\mu \mathrm{m})$ at $913 \mathrm{~K}$. It is clear from this figure that a continuous layer of allotriomorphic ferrite surrounds the austenite grains ( $\xi_{\alpha}=0.10$ ), whereas a small amount of idiomorphic ferrite is found inside the austenite grains $\left(\xi_{I D I}=0.02\right)$. By contrast, the microstructure obtained after $80 \mathrm{~s}$ (Fig. 4(b)) shows almost the same amount of allotriomorphic ferrite ( $\xi_{\alpha}=0.12$ ), whereas a substantial increase in the amount of idiomorphic ferrite ( $\left.\xi_{I D I}=0.06\right)$ is observed. Likewise, it is observed in Fig. 3(a) that the value of $\xi^{*}$ at the maximum is higher as temperature decreases which indicates that the ratio between allotriomorphic and idiomorphic ferrite volume fractions is higher at $873 \mathrm{~K}$ than that at 
$913 \mathrm{~K}$. However, after the maximum, $\xi^{*}$ decreases faster at $873 \mathrm{~K}$ than at $913 \mathrm{~K}$ as the slope in Fig. 3(a) and 3(b) indicates.

As idiomorphic ferrite transformation proceeds, $\xi^{*}$ decreases until reach saturation, i.e. a constant value of $\xi^{*}=1$. A minimum value of $\xi^{*}=1$ demonstrates that idiomorphic ferrite always reaches equilibrium later than allotriomorphic ferrite, which occurs at both studied temperatures (Fig. 3(b)). The time required for saturation is longer at $873 \mathrm{~K}$ than that at $913 \mathrm{~K}$ since the total austenite decomposition takes more time as temperature decreases.

The role of PAGS on the competitive allotriomorphic and idiomorphic ferrite transformation is presented in Fig. 5. It is clear from this figure that a coarse PAGS promotes the intragranular formation of ferrite to the detriment of that nucleated on the grain boundaries. This is consistent with the fact that as PAGS increases, the number of nucleation sites inside the austenite grain increases and those on the grain boundary decreases (Fig. 2). The value of $\xi^{*}$ decreases as the austenite decomposition progresses. It is worthy to mention that it is necessary more time to reach saturation as PAGS decreases.

\section{Conclusions}

The allotriomorphic and idiomorphic ferrite competitive process in a medium carbon vanadium-titanium microalloyed steel has been analysed in this work. It could be concluded that idiomorphic ferrite is formed instead of allotriomorphic ferrite, as the 
density of intragranular nucleation sites is in the order of that of the grain boundary nucleation sites. Thus, as PAGS increases, idiomorphic ferrite formation is promoted.

Finally, since both types of proeutectoid ferrite could affect the subsequent transformation of the steel, it is necessary to separately analyse the kinetics of the isothermal formation of allotriomorphic and idiomorphic ferrite. The consideration of proeutectoid ferrite (allotriomorphic + idiomorphic) as a single transformation might lead to wrong conclusions in an analysis of the isothermal decomposition of austenite.

\section{Acknowledgements}

The authors acknowledge financial support from the Spanish Comisión Interministerial de Ciencia y Tecnología (CICYT) (project-PETRI 95-0089-OP). GSB Acero S.A and CEIT are thanked for providing the steel and their collaboration in this project. One of the authors, F.G. Caballero, is grateful to the Consejería de Educación y Cultura de la Comunidad de Madrid-Spain for providing a postdoctoral grant. 


\section{References}

1. C.A. DUBÉ, H.I. AARONSON and R.F. MEHL: Revue de Metallurgie, 1958, 3, 20110.2. H.I. AARONSON: 'Symposium on the Mechanical Properties of Phase Transformations in Metals’, 47; 1955, London, The Institute of Materials.

3. F. ISHIKAWA, T. TAKAHASHI and T. OCHI: Metall. Trans. A, 1994, 25A, 929-36.

4. K. YAMAMOTO, S. MATSUDA, T. HAZE, R. CHIJIIWA and H. MIMURA: in 'Symposium on Residual and Unspecified Elements in Steel', 266-84; 1989, Philadelphia, PA, ASTM.

5. T. OCHI, T. TAKAHASHI and H. HARADA: Iron and Steelmaker, 1989, 16, 21-8.

6. R.A. FARRAR and P.L. HARRISON: J. Mater. Sci., 1987, 22, 3812-20.

7. M. IMAGUMBAI, R. CHIJIIWA, N. AIKAWA, M. NAGUMO, H. HOMMA, S. MATSUDA and H. MIMURA: in 'HSLA Steels: Metallurgy and Applications', (ed. J.M. Gray), 557-66; 1985, Metals Park, OH, ASM International.

8. D.J. ABSON and R.J. PARETER: Int. Met. Rev., 1986, 31, 141-94.

9. H.K.D.H. BHADESHIA: 'Bainite in Steels', 245-90; 1992, London, The Institute of Materials.

10. M.A. LINAZA, J.L. ROMERO, J.M. RODRIGUEZ-IBABE and J.J. URCOLA: Scripta Metall. Mater, 1993, 32, 395-400.

11. F. ISHIKAWA and T. TAKAHASHI: Iron Steel Inst. Jpn. Int., 1995, 35, 1128-33.

12. M.A. LINAZA, J.L. ROMERO, J.M. RODRÍGUEZ-IBABE and J.J. URCOLA: Scripta Metall., 1993, 29, 217-22.

13. C. CAPDEVILA, F.G. CABALleRO and C. GARCÍA DE ANDRÉS: Metall. Mater. Trans. A, 2001, 32A, 661-9. 
14. C. CAPDEVILA, F.G. CABALLERO and C. GARCIA DE ANDRES: Scripta Metall. Mater., 2001, 44, 593-600.

15. C. CAPDEVILA, F.G. CABALLERO and C. GARCIA DE ANDRES: Scripta Metall. Mater., 2001, 44, 129-34.

16. C. CAPDEVILA, F.G. CABALlERO and C. GARCÍA DE ANDRÉS: Metall. Mater. Trans. A, 2001, 32A, 1591-7.

17. C. GARCÍA DE ANDRÉS, G. CARUANA, and L.F. ALVAREZ: Mater. Sci. Eng., 1998, A241, 211-215.

18. G.F. VANDER VOORT: 'Metallography. Principles and Practice', 427; 1984, NY, McGraw-Hill Book Company.

19. A.O. KLUKEN and O. GRONG: Metall. Mater. Trans. A, 1989, 20A, 1335-1349.

20. O. GRONG: in 'Metallurgical Modelling of Welding', (ed. H.K.D.H. Bhadeshia) 415; 1997, London, Institute of Materials.

21. J.W. CHRISTIAN: 'Theory of transformations in Metals and Alloys', 2nd edition, 476; 1975, Oxford, Pergamon Press.

22. J. W. CAHN: Acta Metall., 1956, 4, 449-59.

23. R.H. SILLER and R.B. MCLELLAN: Metall. Trans., 1970, 1, 985-94.

24. H.K.D.H. BHADESHIA: Metal Sci., 1981, 15, 477-9.

25. S.S. BABU and H.K.D.H. BHADESHIA: J. Mat. Sci. Lett., 1995, 14, 314-6.

26. R.C. REED, and H. K. D. H. BHADESHIA: Mater. Sci. Technol., 1992, 8, 421-35.

27. J.R. BRADLEY, J.M. RIGSBEE and H.I. AARONSON: Metall. Trans. A, 1977, $\mathbf{8}^{\mathbf{a}}$, $323-31$. 
28. J. W. CHRISTIAN: 'Theory of transformations in Metals and Alloys', 2nd edition, 482; 1975, Oxford, Pergamon Press.

29. H.K.D.H. BHADESHIA: Progress in Materials Science, 1985, 29, 321-372.

30. G.J. SHIFLET, J.R. BRADLEY and H.I. AARONSON: Metall. Trans. A, 1978; 9A, 999-1005.

31. W.F. LANGE, M. ENOMOTO and H.I. AARONSON: Metall. Trans. A, 1988, 19A, $427-40$. 
Table 1. Chemical Composition (mass-\%)

\begin{tabular}{ccccccccccc}
\hline $\mathrm{C}$ & $\mathrm{Si}$ & $\mathrm{Mn}$ & $\mathrm{Cr}$ & $\mathrm{Al}$ & $\mathrm{Ti}$ & $\mathrm{V}$ & $\mathrm{Cu}$ & $\mathrm{O}$ & $\mathrm{Mo}$ & $\mathrm{S}$ \\
\hline 0.37 & 0.56 & 1.45 & 0.04 & 0.024 & 0.015 & 0.11 & 0.14 & 0.004 & 0.025 & 0.043 \\
\hline
\end{tabular}


Table 2. Austenitising and Isothermal Parameters

\begin{tabular}{ccc}
\hline Austenitisation Temperature & PAGS & Isothermal Temperature \\
$(\mathrm{K})$ & $(\mu \mathrm{m})$ & $(\mathrm{K})$ \\
\hline 1523 & 76 & 913 \\
1273 & 11 & 873 \\
\hline
\end{tabular}


Table 3. Calculated Values of $C^{\alpha \gamma}, C^{\gamma \alpha}, \xi(\theta), D_{C}^{\gamma}, \alpha_{1}$, and $\alpha_{3}$ at the Two Different

Studied Temperatures

\begin{tabular}{ccccccc}
\hline$T$ & $C^{\alpha \gamma}$ & $C^{\gamma \alpha}$ & $\xi(\theta)$ & $D_{C}^{\gamma} \times 10^{-14}$ & $\alpha_{1} \times 10^{-7}$ & $\alpha_{3} \times 10^{-7}$ \\
$(\mathrm{~K})$ & $($ wt.-\%) & $($ wt.-\%) & & $\left(\mathrm{m}^{2} \mathrm{~s}^{-1 / 2}\right)$ & $\left(\mathrm{m} \mathrm{s}^{-1 / 2}\right)$ & $\left(\mathrm{m} \mathrm{s}^{-1 / 2}\right)$ \\
\hline 873 & 0.016 & 1.195 & 0.023 & 5.8 & 4.8 & 5.2 \\
913 & 0.016 & 0.903 & 0.016 & 12.0 & 4.9 & 6.0 \\
\hline
\end{tabular}


Table 4. Calculated Values of $\alpha_{1}, \phi, \xi_{\alpha}^{E Q}$ and $S_{V}^{G B}$ at Two Different

\section{Temperatures}

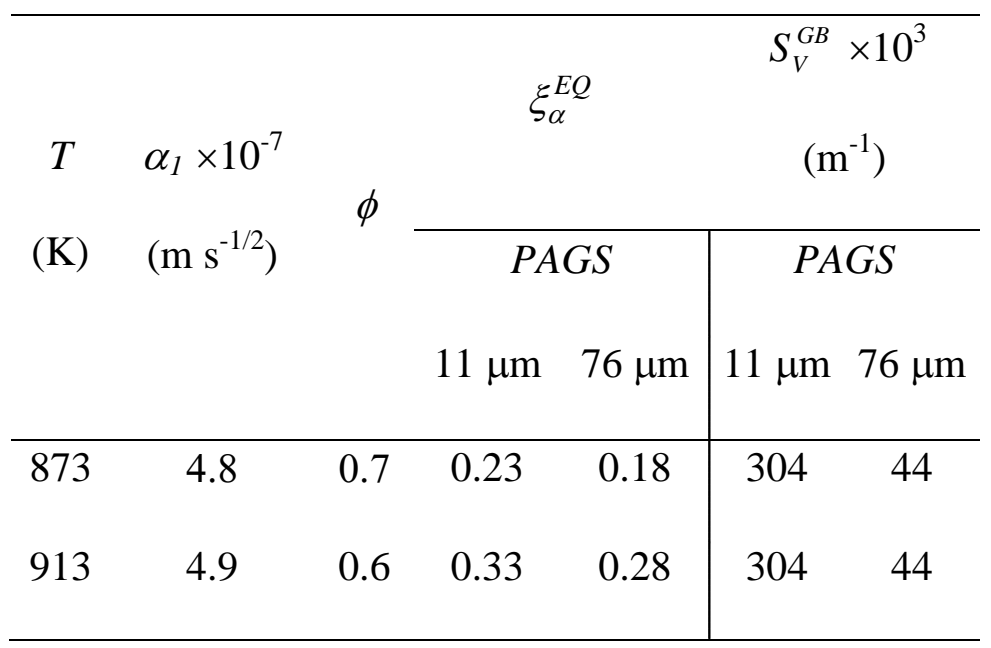


Table 5. Calculated Values of $\boldsymbol{I}_{I D I}, \alpha_{3}, \xi_{I D I}^{E Q}$ and $S_{V}^{I N C}$ at Two Different

Temperatures

\begin{tabular}{|c|c|c|c|c|c|c|}
\hline \multirow{3}{*}{$\begin{array}{c}T \\
(\mathrm{~K})\end{array}$} & \multirow{3}{*}{$\begin{array}{l}I_{I D I} \times 10^{8} \\
\left(\mathrm{~m}^{-2} \mathrm{~s}^{-1}\right)\end{array}$} & \multirow{3}{*}{$\begin{array}{l}\alpha_{3} \times 10^{-7} \\
\left(\mathrm{~m} \mathrm{~s}^{-1 / 2}\right)\end{array}$} & \multicolumn{4}{|c|}{$\begin{array}{c}S_{V}^{I N C} \times 10^{3} \\
\left(\mathrm{~m}^{-1}\right)\end{array}$} \\
\hline & & & \multicolumn{2}{|c|}{ PAGS } & \multicolumn{2}{|c|}{ PAGS } \\
\hline & & & $11 \mu \mathrm{m}$ & $76 \mu \mathrm{m}$ & $11 \mu \mathrm{m}$ & $76 \mu \mathrm{m}$ \\
\hline 873 & 1.2 & 5.2 & $<0.01$ & 0.06 & 11 & 76 \\
\hline 913 & 5.5 & 6.0 & $<0.02$ & 0.08 & 11 & 76 \\
\hline
\end{tabular}



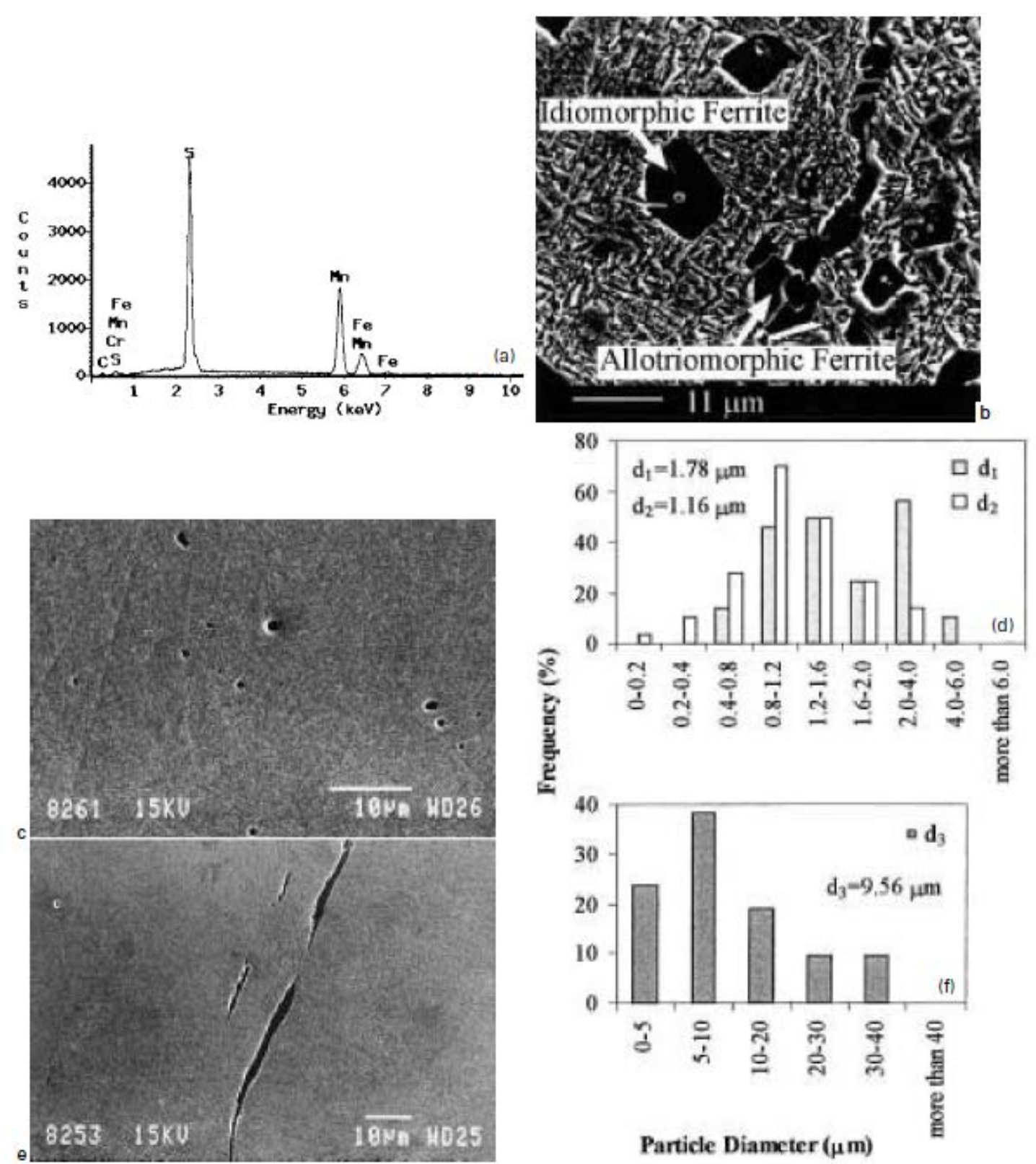

(a) nature, morphology and size distribution in (b) transversal and (c) longitudinal section to rolling direction

1 SEM analysis of the steel inclusions. 


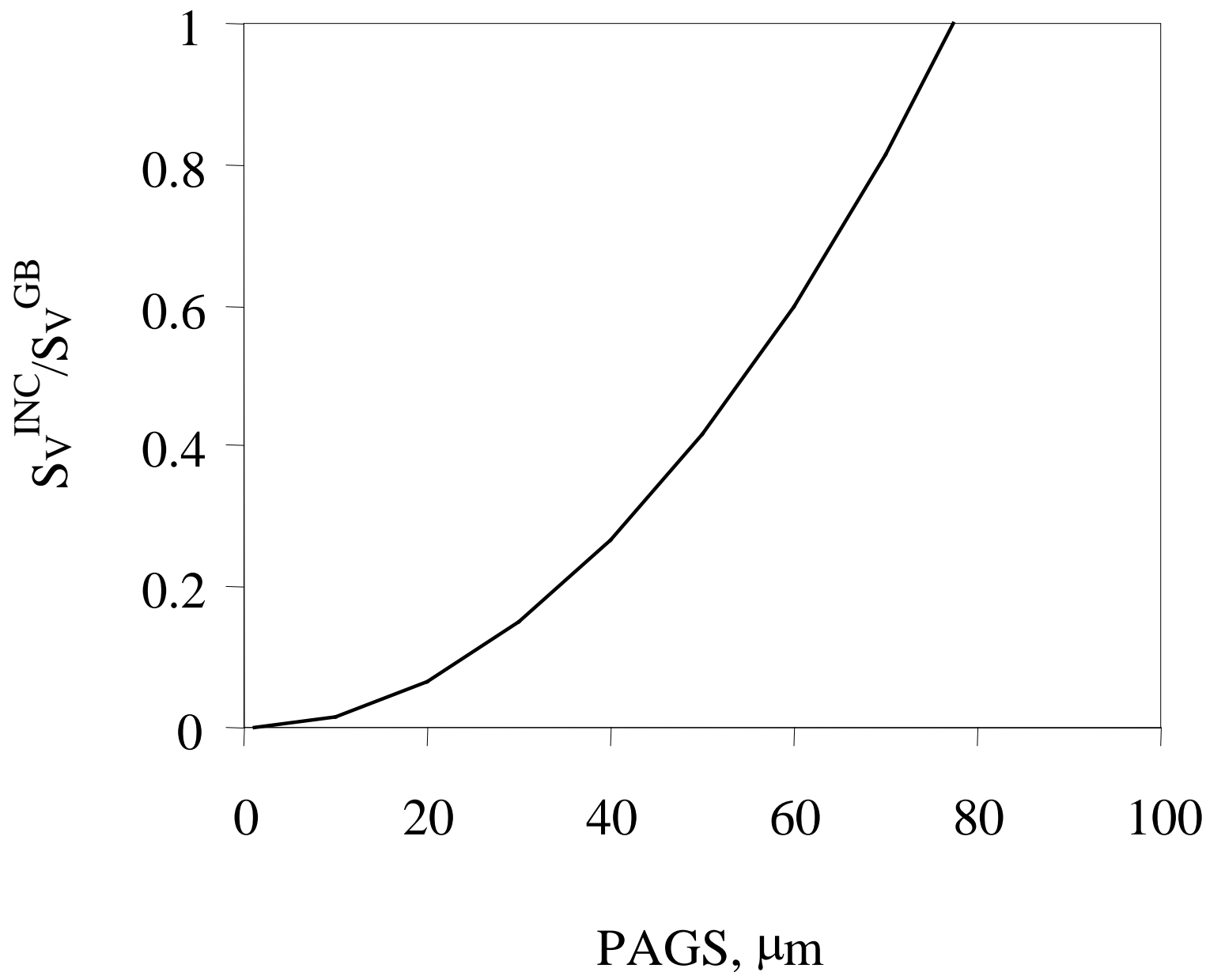

2 Evolution of the ratio between intragranular and grain boundary nucleation sites as a function of the PAGS.. 


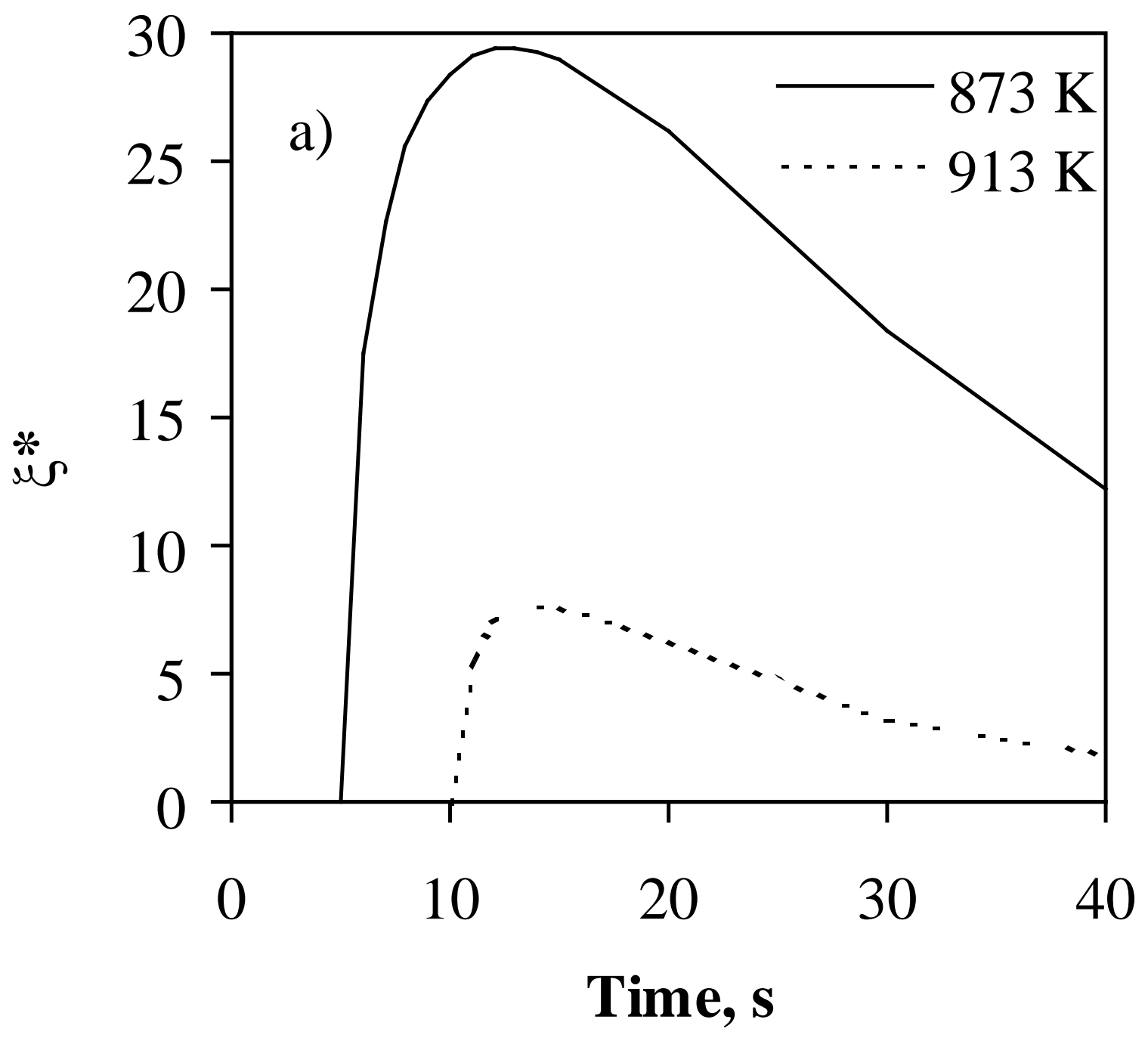

(a) 


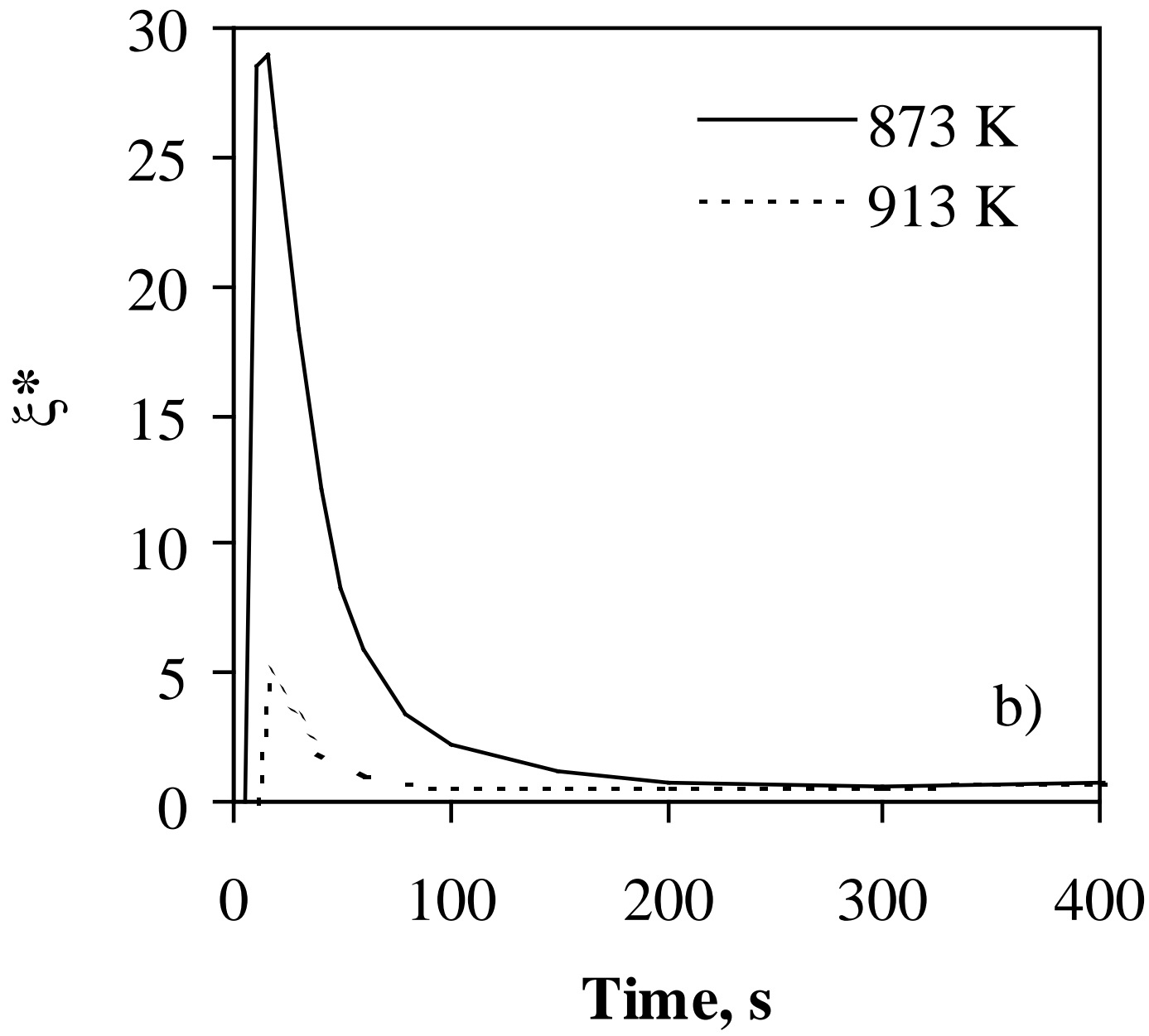

(b)

(a) during the early stages of transformation; and (b) until $\xi^{*}$ reaches saturation.

3 Evolution of the calculated values of $\xi^{*}$ as a function of temperature for a PAGS of $76 \mu \mathrm{m}:$ 


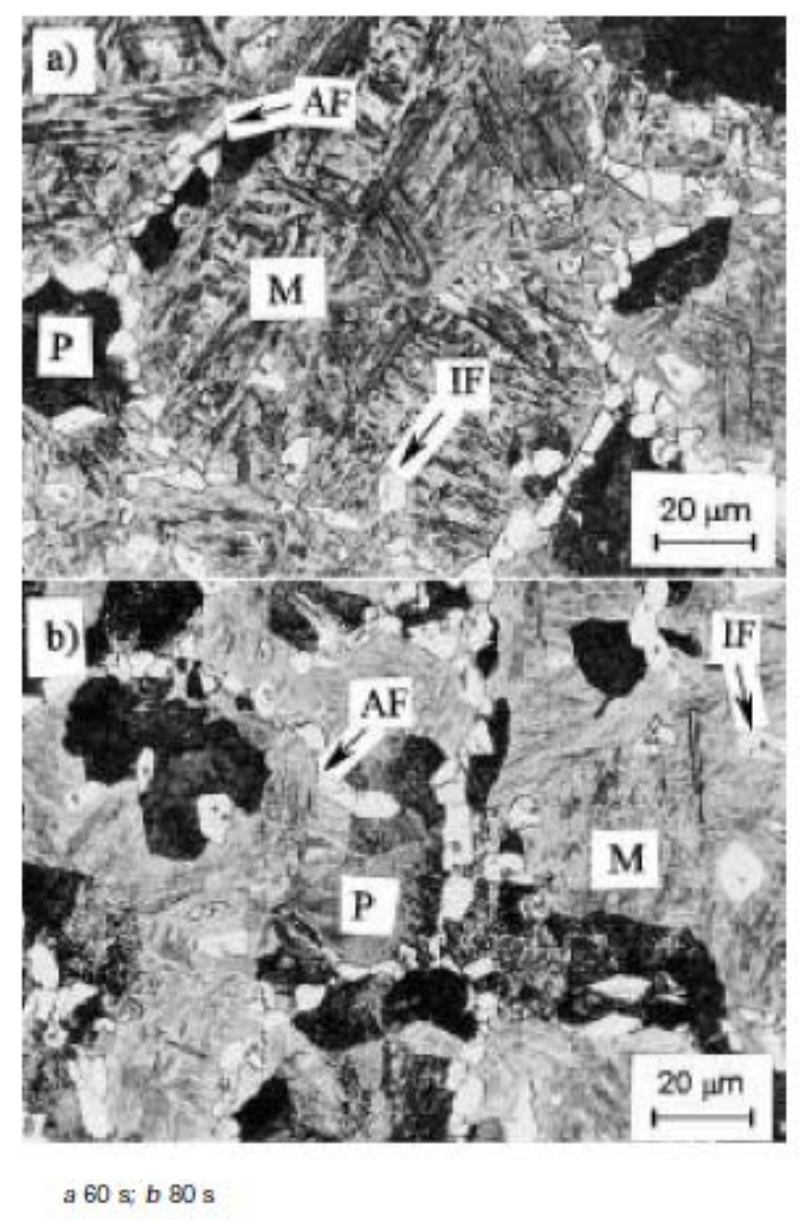

(a) $60 \mathrm{~s}$ and (b) $80 \mathrm{~s}$. (AF = Allotriomorphic ferrite, IF = Idiomorphic Ferrite, $\mathrm{P}=$ Pearlite and $\mathrm{M}=$ Martensite).

4 Microstructures obtained by isothermal decomposition of austenite (PAGS of 76 $\mu \mathrm{m})$ at $913 \mathrm{~K}$ 


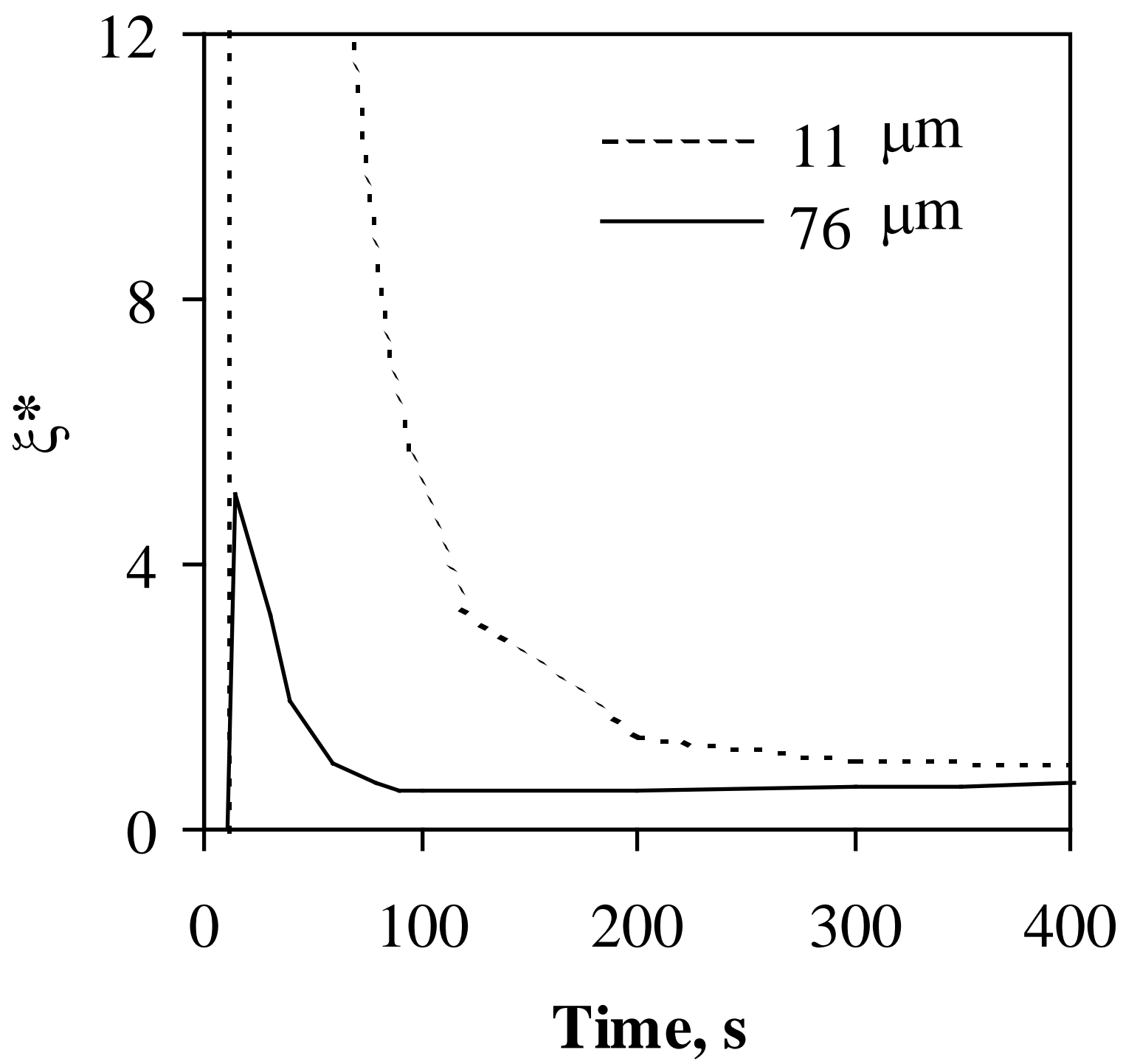

5 Evolution of the calculated values of $\xi^{*}$ as a function of PAGS for a temperature of $913 \mathrm{~K}$. 